\title{
Irina Gruschewaja - Frau Europas 2011
}

\author{
Verleihung im 20. Jahr des Preises „Frau Europas“ am 24. November 2011 in Berlin
}

\section{Katharina Wolf}

Delegierte des djb im Vorstand der EBD, Berlin

Der „Preis Frauen Europas - Deutschland“ feierte am 24. November 2011 sein 20-jähriges Jubiläum: Seit 1991 verleiht das Netzwerk Europäische Bewegung Deutschland (EBD) diese Auszeichnung an Frauen, die sich durch ihr ehrenamtliches gesellschaftliches Engagement für das Zusammenwachsen und die Festigung eines vereinten Europas einsetzen. Ursula Schleicher und Inge Bell stellten rückblickend den Preis und die Preisträgerinnen vor.

Mit Irina Gruschewaja wurde die „Frau Europas 2011“ in Berlin geehrt. Die Bürgerinitiative „Den Kindern von Tschernobyl“, die sie 1989 gemeinsam mit ihrem Mann gründete, hat Auslandsreisen für Kinder aus verstrahlten Gebieten organisiert und seitdem mehr als 500.000 Tschernobyl-Kindern Erholungs- und Gesundungsreisen nach Westeuropa ermöglicht.

Prof. Ursula Männle, Vorsitzende des Ausschusses für Bundes- und Europaangelegenheiten im Bayerischen Landtag und Mitglied im Vorstand der EBD, begrüßte die Jubiläumsveranstaltung eröffnend die vielen anwesenden „Frauen Europas“. Erkennbar sind diese an ihrem „symbolischen Preis, dem sichtbaren Zeichen ihres Engagements“, der blau-goldenen Brosche.

\section{Im Wortlaut:}

\section{Kurze Entstehungsgeschichte}

\section{Ursula Schleicher}

Vizepräsidentin des EP a.D.

Aus Belgien kam in den Achtzigerjahren der Ruf. Eine Abgeordnete im Belgischen Senat - Angèle Verdin - erkannte, dass es unüblich ist, Frauen auszuzeichnen. Damals - obwohl schon über 30 Jahre Geschichte der EG - waren hohe Auszeichnungen und Ehrungen meistens an hohe Ämter geknüpft, d.h. für Frauen Fehlanzeige. Und das, obwohl gerade die Hoffnung auf Frieden in einem gemeinsamen Europa Frauen mehr motivierte als Männer. Es gab bis zu diesem Zeitpunkt kaum einen internationalen Preis, der auch Frauen verliehen worden wäre (Nobel-Preis Marie Curie).

Eine Anfrage bei der EU-Kommission mit der Bitte um Förderung verhalf Angèle Verdin zum Durchbruch ihrer Idee, einen Preis Frauen für Europa zu kreieren. Der Auftrag war: Frauen auszuzeichnen „die grenzüberschreitend in Ehrenamt und Eigeninitiative der Integration Europas dienen und somit auch Vorbild für jede Frau sein könnten“. Nicht die Ranghöhe

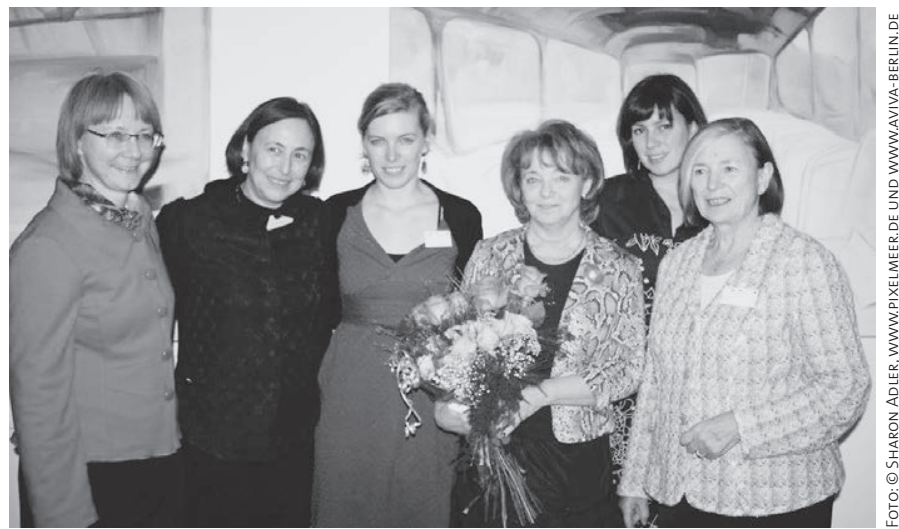

$\Delta$ V.I.n.r.: Katharina Wolf (djb/Vorstand EBD), Marie-Therese Huppertz (SAP - Sponsoren der Preisverleihung), Katrin Lange (EBD), Irina Gruschewaja (Preisträgerin), Karoline Münz (EBD), Prof. Ursula Männle (CSU/Vorstand EBD).

Inge Bell - selbst eine Preisträgerin - stellte in einem Film die bisherigen Preisträgerinnen u.a. Necla Kelek (2008), Gesine Schwan (2005), Dagmar Schipanski (2000) oder Monika Hauser (1995) vor. Ein weiterer Film erläuterte die Arbeit der Preisträgerin 2011.

Wie es zur Brosche und zu anderen zentralen Elementen des Preises Frauen Europas kam, veranschaulichte Ursula Schleicher, Vizepräsidentin des Europäischen Parlamentes a.D.

der auszuzeichnenden Person soll Ausschlag geben, sondern der tatsächliche Einsatz.

Heute - nach 20 Jahren - ist festzustellen: Die Veranstaltungen in Deutschland, zunächst in Bonn (in der Redoute) später in Berlin am Gendarmenmarkt, wurden mit großem Interesse aufgenommen, 300 bis 500 Gäste kamen. Die Europäische Bewegung bekam großen Auftrieb.

Das Engagement der Frauen in und für Europa ist ungebrochen. Es ist schier unglaublich, mit welcher Tatkraft Frauen Einsatz in Europa leisten. Es war in der Jury gar nicht so einfach, Entscheidungen zu treffen, da alle Vorschläge mit unterschiedlichsten Aktivitäten herausragende Ziele verfolgten. Diese Frauen zählen für mich zu den Pionieren Europas. Sie haben bewiesen, dass Eigeninitiativen einen gewinnbringenden Prozess einleiten, der die Europäer einander näher bringt und auf eine friedliche Zukunft hoffen lässt.

Europa steht bereits im zweiten Jahrzehnt des 21. Jahrhunderts. Heftige Diskussionen um die Fortführung sind ausgebrochen. Zweifel und Hoffnungslosigkeit breiten sich aus. Dies ist keine gute Grundlage für die Zukunft. Nehmen wir uns ein Beispiel an diesen vielen Frauen. Sie sind Hoffnungsträger für Europa. Wägen wir nicht ab, was bringt uns Europa, sondern was ist es uns wert! 
„Man darf nie müde werden, Mensch zu sein“"

Irina Gruschewaja, „Frau Europas 2011“

Ich bedanke mich sehr für diese Auszeichnung, weil sie uns und mir zeigt: Unsere Tschernobyl-Kinder sind europäische Menschen, sie sind auch Bürger Europas, wenn auch nicht der Europäischen Union angehörig. Aber dieser Zaun, der zwischen Polen und Belarus steht, ist wie der Eiserne Vorhang zu der Zeit von Stalin: Dieser Zaun trennt uns, und lange Zeit haben wir gedacht, er würde uns einfach abschotten. Das ist aber nicht passiert, weil wir so viele Kinder als Freunde, als Brückenbauer, geschickt haben mit der Botschaft: Ich will leben! Ich will dazugehören!

\section{Die Frage des Menschwerdens ist das $\mathrm{A}$ und $\mathrm{O}$ in Belarus}

(...) Ich fühle mich als werdender Mensch, und ich wünsche mir, dass Sie alle dieses Gefühl in sich entdecken, dass wir nie müde werden, Mensch zu sein. An dieser Stelle bedanke ich mich sehr bei der lieben Rita Süssmuth, Sie haben mir [mit ihrer Laudatio; Anm. d. Red.] aus dem Herzen gesprochen, denn dieses Thema treibt mich seit langem um: Wie viel Mensch ist in uns, wann beginnt der Mensch in uns, und wann endet er? Die Geschichte hat gezeigt, dass es sehr leicht ist, den Menschen aus uns auszutreiben. (...)

(...) Das ist ein Bild, das sehr viele Facetten hat, denn in vielen der weit entlegenen Dörfern, wo die Deutschen die Tschernobyl-Kinder besuchen, sind die Wunden des Krieges nicht vergessen worden. Aber: Dass auch Deutsche gekommen sind, die den Frieden gebracht haben, die die Sorge um unsere Kinder gebracht haben - das ist die internationale Zivilgesellschaft im Einsatz. Das ist die Größe dieses Werkes, das wir alle zusammen aufbauen konnten. „Hilfswerk“ will ich es gar nicht nennen: Das ist kein Hilfswerk, es ist ein Werk der Menschen, die ihr Menschsein erkannt haben. (...)

\section{Lukaschenko erklärte Tschernobyl im Jahr 2000 für über- wunden}

(...) Aber: Nach einer expandierenden Aktion der Hilfe, der Zusammenarbeit, des Wachwerdens der Bürger kam die Zeit - in Deutschland ist der „Ausstieg aus dem Ausstieg“ dafür exemplarisch -, wo Tschernobyl plötzlich kein Thema mehr war. Für Belarus galt das schon lange, weil Lukaschenko bereits im Jahr 2000 erklärte: „Mit Tschernobyl sind wir fertig.“ Und wir mit unserem Engagement, das wir immer politisch verstanden hatten, wenn es auch um Hilfe ging (wir meinten, unser soziales Engagement ist Ausdruck des Ungehorsams, weil in einem paternalistischen Staat, der alles kontrolliert, kein Engagement genehm ist und jedes Engagement den Glauben an die Allmacht des Staates untergräbt) - wir dachten, es ist aus, und wir werden ins Archiv abgeschoben mit unserem Engagement, das politische Folgen hätte haben sollen. Wir wollten damals unsere Zukunft nicht den Politikern allein überlassen. Wir wollten aktive Menschen bleiben, aber wie kann man aktiv sein, wenn man auch arbeiten muss? (...)

(...) 2008, am 13. Oktober, das Dekret 555: Kein Kind darf mehr reisen. Der Hohn der Geschichte: Am selben Tag entscheiden Europarat und Europäisches Parlament, dass Lukaschenko mit seinen Helfershelfern nach Europa einreisen kann. Die Schreie der Kinder, die im darauffolgenden Sommer 2009 hinter dem Zaun geblieben sind, wurden kaum gehört. Wir haben nicht aufgegeben. (...) Wir haben es geschafft: Die Kinder konnten ein Jahr später wieder ins Ausland zu ihren Freunden fahren. Aber natürlich gab es Verluste, weil viele Initiativen vor Ort, die ein Jahr ausgesetzt hatten, ihr Engagement oft nicht mehr fortsetzen konnten. (...)

\section{... und dann kam Fukushima}

Die Geschichte der „Kinder von Tschernobyl“ gegen die Atomkraft wäre vielleicht schon im Archiv gelandet - doch dann kam der furchtbare Schock und ein neues, schreckliches Ereignis, das uns alle wieder wachgerüttelt hat: Fukushima. Und mit Fukushima kam wieder Kraft, die Resignation musste zurückweichen. Wir haben uns wieder zusammengefunden, haben an den Kundgebungen teilgenommen, haben neue Menschen getroffen und engagiert, weil - und das ist eine wichtige Erkenntnis - wir nicht mehr verkraften können: Die Menschheit kann kein Tschernobyl und kein Fukushima mehr verkraften.

Es ist ein sehr, sehr schwerer Weg, den wir alle gehen. Wir dürfen uns nicht beruhigen! Durch unsere Aktivitäten ist bei abertausenden Menschen ein neues Bewusstsein entstanden. (...) Und ich bedanke mich sehr, dass dieser Preis uns gefunden hat in Deutschland, wo wir noch Kraft haben, Mut und Hoffnung.'

\section{Hoffnung ins Land der Hoffnungslosigkeit bringen}

Meine vielen Referate, Reden, Vorträge habe ich „Die Hoffnung im Land der Hoffnungslosigkeit“ genannt. Ich kann nichts weiter sagen als: Die Hoffnung in der Welt der Hoffnungslosigkeit - wenn wir die Welt aus der Perspektive der Gewalt und der Ungerechtigkeit betrachten -, die Hoffnung sind wir! Und die Hoffnung ist im Gegensatz zum Optimismus nicht die Gewissheit, dass alles gut ausgeht, sondern das Engagement in der Gewissheit, dass es sich lohnt, egal wie es ausgeht. Diese Worte von Václav Havel müssen uns beflügeln und ermutigen. Sie müssen in uns den Menschen unterstützen, der wach geworden ist, und der sich nicht abgibt mit dem, was ist.

\section{Quellen:}

http://www.europaeische-bewegung.de/news/man-darf-nie-muedewerden-mensch-zu-sein-irina-gruschewaja-als-frau-europas-2011-ge/ (Zugriff: 23.9.2012)

http://www.europaeische-bewegung.de/aktivitaeten-projekte/preisfrauen-europas/2011-irina-gruschewaja/ (Zugriff: 23.9.2012)

http://www.europaeische-bewegung.de/aktivitaeten-projekte/preisfrauen-europas/preistraegerinnen-1991-2011/ (Zugriff: 23.9.2012)

1 Auszug aus der Rede der Preisträgerin. 
und geistige „Mutter des Preises“, in ihrem Rückblick auf die vergangenen 20 Jahre. Frauen auszuzeichnen, die grenzüberschreitend und in Eigeninitiative Einsatz für Europa zeigen und damit Vorbild für alle Frauen Europas sein können, sei seit 1991 das Anliegen des Preises. Angefangen mit Csilla von Boeselager, seien in den letzten 20 Jahren Frauen wie zum Beispiel Christine Grotensohn, Lea Ackermann, Gudrun SchmidtKärner, Ludmilla Irmscher und Cathrin Schauer mit einer enormen Bandbreite an Projekten und Themen ausgezeichnet worden. Gemeinsam sei allen „Frauen Europas“ ihr Pioniergeist, der auf ganz unterschiedliche Art dazu beitrage, die Bürger Europas einander näher zu bringen.

Laudatorin der „Frau Europas 2011“ war die ehemalige Bundestagspräsidentin Prof. Dr. Rita Süssmuth. Die Ehrenpräsidentin der EBD würdigte Gruschewajas Kampf gegen das Schweigen über die Tschernobyl-Katastrophe in Belarus als vorbildlich auch für Demokratien, in denen die öffentliche Diskussion von Unbequemem gern auf später verschoben werde. Unermüdlich und mit großer Ehrenhaftigkeit habe sich Gruschewaja dafür eingesetzt, dass in und über Belarus hinaus geredet statt geschwiegen wurde - und damit den Nährboden für Veränderung bereitet: „Wenn wir nicht mehr miteinander reden, haben wir keine Chance mehr, etwas zu verändern!“ Um den „Preis Frauen Europas - Deutschland“ für die Zukunft zu sichern, rief Süssmuth die Mitgliedsorganisationen der EBD dazu auf, sich in Zukunft für den Preis einzusetzen.

In ihrer Rede beschwor Irina Gruschewaja, Menschlichkeit und soziales Miteinander als Basis des Zusammenlebens nicht zu vergessen. Man dürfe nie müde werden, Mensch zu sein - auch wenn und grade weil die Geschichte immer wieder gezeigt habe, "wie leicht es ist, den Menschen aus uns auszutreiben“. Für das Menschsein sei die internationale Zivilgesellschaft im Einsatz - ihre Initiative „Den Kindern

\section{Save the Date / Bitte Termin notieren}

Der djb wird bei seinem Empfang anlässlich des Deutschen Juristentages am 19. September 2012 in München die Preisverleihung „Frau Europas 2012“" ausrichten.

von Tschernobyl“" sei deshalb kein Hilfs-, sondern vielmehr ein Menschenwerk. Dessen soziales Engagement mache verdächtig: 2008 erließ Belarus ein Dekret, nach dem kein Kind mehr in Ausland reisen dürfe. Nur auf Druck der Partner in Deutschland, der Schweiz und anderen Ländern sei das Reisen für Kinder ein Jahr später wieder möglich geworden. Die Tschernobyl-Bewegung habe dies nachhaltig geschwächt, erst die Katastrophe von Fukushima habe alle wieder wachgerüttelt. „Wir dürfen uns nicht beruhigen“, beschwor Gruschewa$j a$ das Publikum, noch einen Super-GAU vertrage die Menschheit nicht. Dank der Kinderreisen - 500.000 sind in den vergangenen 21 Jahren nach Deutschland gereist - wüssten nun Millionen Menschen, was es bedeutet, Opfer zu sein und was es bedeutet, Mut zu haben.

Übergeben wurde der Preis von Irina Prinzessin zu SaynWittgenstein, Präsidentin des Preises und selbst Preisträgerin von 1994, die einen weiteren Bogen zur Bedeutung des Preises für das heutige Europa spannte. „Europa hat sich verändert. Die Auszeichnung zeigt, dass wir uns jeden Tag bewusst sein müssen, dass wir uns das alles erobert haben“, gab sie den knapp 180 anwesenden Gästen mit auf den Weg.

Der Empfang im Anschluss an die Preisverleihung bot Gästen, Organisator/inn/en und der Preisträgerin die Möglichkeit, bei einem Glas Wein den erfolgreichen Abend ausklingen zu lassen.

\section{„Frauen in die Roten Roben“ - eine erste Bilanz}

\section{Eva Schübel \\ Vizepräsidentin des djb, Bundesanwältin beim BGH, Karlsruhe}

Die Richterwahl am 29. März 2012 brachte 25 Prozent der vorgeschlagenen Richterinnen in die dritte Instanz und lässt sich mit einer Besetzungsquote von 30 Prozent sehen. Von den 12 offenen Stellen am Bundesgerichtshof sind zwar nur zwei mit Frauen besetzt worden. Zum Bundesverwaltungsgericht kommen aber zwei neue Richterinnen und ein neuer Richter. Der Bundesfinanzhof wird neben einem Richter mit einer Bundesrichterin verstärkt. Für das Bundesarbeitsgericht und das Bundessozialgericht fanden in diesem Jahr keine Wahlen statt.

Dieses insgesamt befriedigende Ergebnis konnte erreicht werden, weil unter den Kandidat/inn/en knapp 35 Prozent Richterinnen waren und sich bei früheren Wahlen sehr oft die wenigen vorgeschlagenen Frauen überproportional durchge- setzt hatten. Für den Bundesgerichtshof sind erstmals seit 2007 (die früheren Zahlen sind mir nicht bekannt) ein Drittel Frauen vorgeschlagen worden (13 von $39 \mathrm{Kandidat} / \mathrm{inn} / \mathrm{en})$ und damit zumindest eine Richterin für jede der $12 \mathrm{zu}$ besetzenden Stellen. Dies trifft auch für die drei Bundesrichterstellen am Bundesverwaltungsgericht zu. Auf dessen Vorschlagsliste standen drei Richterinnen und acht Richter. Gegenüber den Zahlen seit 2007 ist die erreichte Quote von 28 Prozent schon sehr gut (zuletzt 2011: eine Frau unter 17). In der Zukunft angekommen ist der Bundesfinanzhof: Für die zwei Stellen sind vier Richterinnen und vier Richter vorgeschlagen worden.

Insgesamt hat die Aktion des djb ihr Ziel, dass für die Richterwahl 2012 mindestens 30 Prozent Frauen vorgeschlagen werden, mit 35 Prozent sogar übertroffen. Diese Zahl ist kein Zufallstreffer, sondern die Frucht unserer umfangreichen Aktivitäten im Vorfeld der Wahl. 